\title{
La situation des Sames du nord dans les communautés d'Enontekiö et de Sodankylä en Finlande
}

Az északi számi helyzete Enontekiö és Sodankylä beszélőközösségeiben, Finnországban

The Northern Sami Situation in the Communities of Enontekiö and Sodankylä (Finland)

\section{Zsuzsa Duray}

Traducteur : Charlotte Karady

\section{(2) OpenEdition Journals}

Édition électronique

URL : https://journals.openedition.org/efo/430

DOI : $10.4000 /$ efo.430

ISSN : 2275-1947

Éditeur

INALCO

Édition imprimée

Date de publication : 1 décembre 2012

ISBN : 978-2-343-02592-6

ISSN : 0071-2051

Référence électronique

Zsuzsa Duray, « La situation des Sames du nord dans les communautés d'Enontekiö et de Sodankylä en Finlande », Études finno-ougriennes [En ligne], 44 | 2012, mis en ligne le 22 janvier 2014, consulté le 10 juillet 2021. URL : http://journals.openedition.org/efo/430 ; DOI : https://doi.org/10.4000/efo.430

Ce document a été généré automatiquement le 10 juillet 2021.

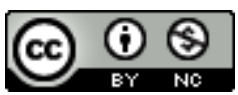

Études finno-ougriennes est mis à disposition selon les termes de la Licence Creative Commons Attribution - Pas d'Utilisation Commerciale 4.0 International. 


\section{La situation des Sames du nord dans les communautés d'Enontekiö et de Sodankylä en Finlande}

Az északi számi helyzete Enontekiö és Sodankylä beszélőközösségeiben, Finnországban

The Northern Sami Situation in the Communities of Enontekiö and Sodankylä (Finland)

Zsuzsa Duray

Traduction : Charlotte Karady

\section{Les lieux de l'étude}

1 La région où j'ai fait mes recherches en 2001-2002 est la Laponie finlandaise, lieu d'habitation officiel et traditionnel de la communauté same de Finlande. La plupart des communautés sames qui y résident, qui sont de plus en plus étroitement rattachées à la langue et à la culture finnoises, utilisent leur langue maternelle dans des situations de plus en plus limitées, compliquant par là même la préservation de cette langue par les générations à venir. Parmi les différents territoires de cette région, j'ai choisi de me concentrer sur les zones autogérées les plus méridionales, celles d'Enontekiö et de Sodankylä (cf. carte) dans la mesure où il n'y a pas encore eu de recherches sur leur situation linguistique ${ }^{2}$. Ces communautés sont plus avancées en matière de changement de langue que, par exemple, celles des territoires plus septentrionaux de la Laponie finlandaise, celles de la région d'Utsjoki, plus fermées et dont les membres sont plus nombreux. C'est grâce à leur nombre et à leur concentration, ainsi qu'au travail des institutions soutenant la langue et la culture sames, que celles-ci bénéficient d'un soutien accru de la part de l'État et que, de ce fait, leurs membres, malgré l'émigration due à l'écroulement de leur statut socio-économique, préservent mieux leur langue. Nous trouvons une situation tout aussi positive que celle d'Utsjoki dans le territoire 
septentrional de la commune d'Inari où, en raison de la concentration des Sames, des programmes de préservation de la langue dès le jardin d'enfants (qui ont obtenu des résultats positifs depuis des années), et du sérieux soutien apporté par l'État aux efforts de préservation de la langue, le changement de langue semble s'être ralenti. J'examine ensemble ces deux territoires en raison de leur similitude en matière de langue, de société, d'histoire et d'autres facteurs.

Les communautés d'Enontekiö et de Sodankylä

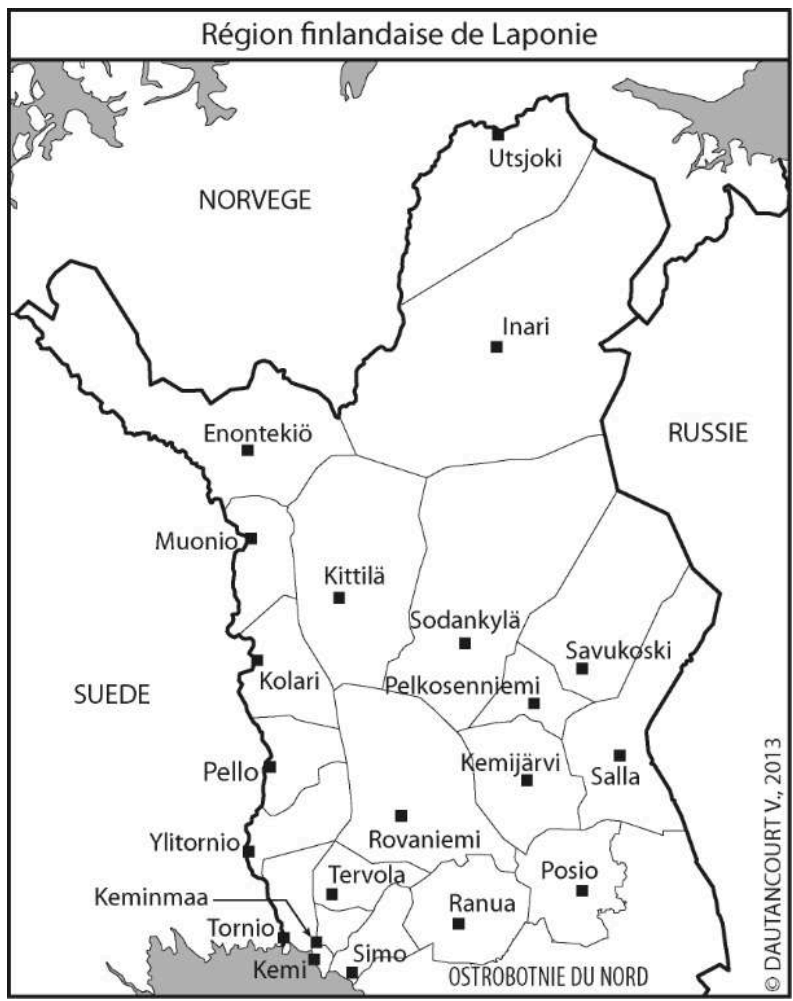

2 Au cours de l'année de ma recherche, en 2002, 176 personnes qui se disaient sames vivaient dans le territoire d'Enontekiö; en 2010, ce chiffre était monté à 187. Le territoire de Sodankylä était habité par 131 personnes l'année de mon terrain, et par 119 en 2010. Dans les trois décennies précédant l'enquête, le nombre des Sames dans le territoire d'Enontekiö était passé de 170 à 187, alors que dans le territoire de Sodankylä leur nombre n'avait cessé de diminuer (cf. www.stat.fi).

Des évolutions de ce type s'expliquent par le fait que ce sont les Sames vivant dans le territoire de Sodankylä, à la frontière méridionale de la Laponie, qui ont toujours entretenu les relations les plus intenses avec les communautés finnoises, majoritaires. Dans ces territoires, les Finnois ont fait prévaloir plus tôt qu'ailleurs leurs tentatives de modernisation, et naturellement celles-ci rejetaient à l'arrière-plan, voire contrecarraient la poursuite des activités relevant du mode de vie traditionnel des Sames (notamment l'élevage des rennes). Ceci a débouché sur la migration des Sames vers les territoires plus au Nord et plus à l'Ouest. La présence de plus en plus importante des Finnois a contribué également à la multiplication des mariages mixtes ; elle a permis de faire passer à l'arrière-plan l'enseignement de la langue minoritaire, ce qui a accéléré à son tour le changement de langue. La proportion des Sames dans la population locale n'est aujourd'hui que d'à peine $1,5 \%$; ils poursuivent l'élevage du 
renne avec des Finnois, sous la direction d'unions d'éleveurs rassemblant Finnois et Sames (paliskunta). La plupart des Sames y ont préservé leur économie de subsistance, bien que celle-ci fournisse des revenus plus bas que les autres professions.

4 Le multilinguisme d'Enontekiö, unique en son genre, tient à la proximité de la Norvège et de la Suède. Dans cette commune, la troisième en Finlande par la taille $\left(8.464 \mathrm{~km}^{2}\right)$, $8 \%$ de la population, chiffre en baisse constante, se disent sames. Une grande partie des communautés qui y résident tirent leurs revenus du tourisme (flux de touristes dans les monts de Laponie par la voie qui relie Enontekiö à la Suède et à la Norvège), et dans une moindre mesure de l'élevage traditionnel du renne. Leur proportion ne baisse donc pas autant qu'à Sodankylä, car là, et encore plus dans les villages le long du Teno, on trouve plusieurs communautés sames qui, en raison de leur isolement, préservent mieux qu'ailleurs leur langue et leur identité. De plus, l'usage de la langue same est favorisé par le fait que les Sames du côté finlandais entretiennent des rapports étroits avec leurs parents samophones du côté norvégien. Pour ceux du côté finlandais, la conscience d'appartenir à la même communauté ethnique et le fait de pouvoir s'exprimer dans la langue commune, facilite les contacts quotidiens et contribue à la préservation de la langue.

\section{La formation de la situation linguistique dans la communauté étudiée}

5 Dans les communautés que j'ai étudiées, ce sont avant tout les transformations sociales et politiques qui ont permis le changement de langue. Jusqu'aux années 1700 , ces communautés utilisaient encore exclusivement les langues sames. Aujourd'hui, dans les parties les plus septentrionales de la Laponie, et là où j'ai fait mon terrain, la norme est le multilinguisme, là où l'histoire a conduit à la formation d'une communauté ethniquement hétérogène. Avant les années 1950, les contacts finno-sames étaient caractérisés par la diglossie, c'est-à-dire que l'usage des langues finnoise et same était distingué par les différents niveaux d'utilisation de la langue, ce qui a longtemps permis la survie du same. Aujourd'hui, le same et la langue majoritaire se trouvent dans une phase d'usage équilibré, alterné, dans une situation de bilinguisme par adjonction, c'est-à-dire que la communauté n'est touchée par aucune stigmatisation ou discrimination de la part de la majorité. On sent cependant une tendance à la perte fonctionnelle de la langue, c'est-à-dire à la diminution progressive des niveaux d'usage de la langue, qui est due à de nombreux facteurs et tout d'abord au fait que la communauté des locuteurs n'est plus en mesure de rester aussi fermée que le demandait le mode de vie traditionnel ; l'environnement linguistique général pèse de plus en plus sur les niveaux de langue formel et informel. En dépit de tout cela, on peut distinguer des facteurs contraires au recul de la langue minoritaire, par exemple la popularité croissante de l'enseignement de la langue maternelle, l'action, de plus en plus intense, d'institutions subventionnées par l'État pour le développement de la langue et pour la défense des intérêts des Sames, les activités liées au mode de vie traditionnel et mettant en avant la préservation de la langue, notamment l'élevage de rennes et l'artisanat, ainsi que les manifestations organisées par les Sames et formant une part importante de la culture same. Dans ces communautés, donc, le changement de langue et la préservation de la langue maternelle sont présents en même temps. 
6 La plupart des habitants de la Laponie ont pour langue maternelle le finnois, plus rarement le same, plus rarement encore le same skol, le norvégien, le suédois ou le russe. Nous pouvons subdiviser les familles en quatre groupes, suivant la langue qu'elles utilisent: (1) celles qui parlent exclusivement finnois, (2) celles qui parlent surtout finnois, et rarement same, (3) celles qui parlent surtout same, et rarement finnois, et (4) celles qui parlent exclusivement same. Ce dernier modèle linguistique caractérise l'interaction des familles sames entre elles, ce qui est de plus en plus rare. Dans la plupart des cas de mariages mixtes, l'usage du same perd de son sens. La communauté d'aujourd'hui est ethniquement mixte, elle se compose de Sames, de Finnois et de descendants de familles finno-sames qui se rattachent, sur la base de la parenté, de la culture ou de la langue, à l'une ou à l'autre de ces communautés.

\section{Les outils de la recherche et les informateurs}

7 J'ai choisi comme instrument de recherche un questionnaire contenant surtout des questions fermées. J'ai choisi les informateurs à partir d'un échantillon préétabli, sur la base de l'âge, du sexe et de la profession. Je suis ainsi arrivée à un échantillon de 225 informateurs potentiels, dont 59 ont finalement été associés à ma recherche.

8 En raison de la diversité des expériences vécues par les membres de la communauté, et en restreignant les limites des trois générations, j'ai subdivisé la communauté étudiée en quatre groupes: les jeunes (âgés de 25 à 40 ans), les gens d'âge moyen (de 41 à55 ans), la génération des personnes d'âge moyen avancé (de 56 à 70 ans) et les personnes âgées (71 ans et plus).

9 La première génération, celle des habitants les plus âgés des villages, est représentée par onze personnes (cinq hommes et six femmes). C'est la génération dont l'une des expériences principales est la déportation qui a suivi la Deuxième Guerre mondiale et la politique d'assimilation forcée qui l'a suivie. Les représentants de cette génération qui ont évité la déportation ou ceux qui sont revenus des territoires méridionaux n'ont pas eu la possibilité d'apprendre à lire et à écrire dans leur langue, beaucoup d'entre eux n'ont pas été scolarisés. La plupart d'entre eux vivaient de l'élevage des rennes, utilisaient leur langue dans le cercle familial, n'entraient pas en relation avec les Finnois et se mariaient avec des Sames.

10 La deuxième génération, celle des personnes d'âge moyen avancé, est représentée par dix-sept personnes (sept hommes et dix femmes), pour qui l'expérience principale est la disparition progressive, après la Deuxième Guerre mondiale, de l'isolement de la communauté, l'immigration massive des Finnois, les possibilités accrues de mobilité, la restriction du champ d'utilisation du same, la généralisation de l'éducation en finnois et la multiplication des mariages mixtes permettant l'avancement social. Ils sont de plus en plus nombreux dans cette classe d'âge à avoir suivi en finnois des études primaires et secondaires. Ceux qui n'ont pas été scolarisés ont continué à vivre de l'élevage des rennes, un grand nombre de ceux qui avaient été scolarisés sont partis chercher des sources de revenus dans les territoires du Sud.

11 La troisième génération, celle des gens d'âge moyen, comprend dix-neuf personnes neuf hommes et dix femmes -, nées pour beaucoup de mariages mixtes, qui ont donc assimilé le finnois comme langue maternelle et qui, dans la plupart des cas, vivent elles-mêmes dans une situation de mariage mixte. Ces personnes sont de plus en plus nombreuses à avoir un diplôme universitaire, mais la plupart des membres de cette 
classe d'âge ont été contraints de quitter leur maison familiale quand ils étaient enfants pour aller étudier dans les écoles et vivre dans les internats des grandes agglomérations de Laponie; ils préfèrent utiliser le finnois, renonçant à leur langue maternelle. Les membres de cette génération, après avoir fini leurs études et suivant les possibilités d'emploi ont quitté leur pays natal et ont construit leur vie dans le Sud de la Finlande ou dans les grandes villes de Laponie.

La quatrième génération est celle des jeunes qui, à l'époque de l'enquête, avaient entre 25 et 40 ans. La plupart d'entre eux sont finnisés, mais ils n'ont pas perdu leur langue. Une partie de cette génération - surtout ceux qui ont fait les études les moins avancées - a été pleinement assimilée dans la culture majoritaire, mais grâce à la revitalisation des mouvements sames, aux institutions nationales qui les soutiennent, aux attitudes positives de la majorité, se considère comme same et s'efforce de maintenir ses traditions. On trouve aussi dans cette génération des gens qui ayant retrouvé leurs racines ethniques, après la fin de leurs études supérieures - souvent en same - retournent dans leur pays natal pour y participer à la revitalisation du same et de la culture same, en enseignant le same par exemple ou encore en travaillant dans les centres de recherche.

Trente-cinq femmes et vingt-quatre hommes ont participé à mon enquête. Pour ce qui est de leur profession, $60 \%$ d'entre eux ( 25 personnes) vivaient de l'élevage des rennes, dont dix-neuf hommes et six femmes qui, en tant qu'épouses d'éleveurs de rennes, avaient des activités en rapport avec cette forme d'élevage. Parmi les informateurs, seulement deux étaient retraités, parce que malgré leur âge, la plupart des membres de la génération d'âge moyen avancé participaient à l'élevage des rennes ou travaillaient dans le tourisme ou encore, dans le cas des femmes, vivaient de la vente des produits de l'artisanat. Dans mon échantillon, il y avait un seul chômeur, quelques enseignants, et davantage d'entrepreneurs travaillant dans la branche des services et représentant $58 \%$ des informateurs. $34 \%$ d'entre eux, après la fin des études primaires, n'avaient pas poursuivi leurs études, alors que les $66 \%$ restants avaient terminé des études secondaires ou supérieures.

Sur les personnes interrogées, $90 \%$ avaient assimilé le same à la maison alors que les $10 \%$ restant, relevant - fait caractéristique - de la jeune génération, avaient appris le same à l'école. $78 \%$ étaient nées et avaient vécu leur enfance et leur jeunesse à Enontekiö ou Sodankylä, les autres provenaient, de même que leurs parents, d'autres régions de Laponie. Les célibataires représentaient $17 \%$; mais quand les personnes interrogées avaient un conjoint ou une conjointe, ceux-ci étaient aussi originaires d'Enontekiö (50\%) ou de Sodankylä ( $20 \%$ ), étaient sames, ou encore originaires de villages finnois, et vivaient également de l'élevage de rennes ( $43 \%)$ ou d'une autre activité, semblable à celle de leur conjoint ou de leur conjointe. Quant aux conjoints, $69 \%$ avaient appris le same à la maison, $10 \%$ à l'école, alors que $21 \%$ ne le parlaient absolument pas. Tous les informateurs étaient bilingues, mais presque la moitié, surtout ceux d'Enontekiö, à proximité de la Suède et de la Norvège, étaient polyglottes. Les membres de la génération d'âge moyen avancé, ainsi que ceux de la partie de la région de Sodankylä la plus proche de la Russie, parlaient russe.

Ma supposition est que les différences dans les pratiques linguistiques entre les quatre groupes d'âge présentés correspondent aux différences de sexe, de profession et de niveau de scolarisation des locuteurs sames du territoire étudié. 


\section{Utilisation de la langue}

16 J'aurais pu essayer de faire appel à différentes manières, différentes méthodes de rassembler l'échantillon dont j'avais besoin - c'est un fait que cela nous aurait donné une image plus claire de l'usage réel de la langue - mais les possibilités de collecte de données limitaient le cercle des méthodes utilisables. Si l'on part du principe que le seul instrument de recherche possible était le formulaire - sans compter les questions posées lors des interviews de test des questionnaires - j'ai essayé de concevoir le questionnaire de telle sorte qu'il reflète, dans la mesure du possible, de la manière la plus fidèle possible les pratiques d'usage de la langue dans la communauté étudiée, et qu'il montre aussi précisément que possible la manière dont ses membres l'utilisent.

17 Sur la base de quelques-unes des unités du questionnaire sur le choix de la langue et sur son usage, je m'efforcerai ci-dessous de montrer quels sont les modèles et les pratiques linguistiques caractéristiques de la communauté au moment de l'étude. Je commencerai par analyser les résultats de la première partie du questionnaire.

Les niveaux qui figurent dans le questionnaire représentent les contextes les plus typiques des interactions de la communauté same. Le premier niveau que j'ai identifié est celui des contacts personnels, où les partenaires les plus caractéristiques de mes informateurs sont les membres de leur famille, leurs camarades d'école, leurs professeurs, leurs voisins, les personnes inconnues et les employés ou fonctionnaires. Naturellement, j'ai voulu savoir quelle langue ou quelles langues les informateurs utilisent au niveau en question.

On voit bien dans le diagramme que, conformément à ce que je supposais, l'usage du same semble restreint au niveau d'un emploi familial. C'est surtout dans la communication avec les parents et avec les frères et sœurs que les informateurs font appel à la langue same. L'interaction entre les informateurs et leurs enfants, ainsi que celle entre conjoints, est caractérisée par l'usage aussi bien du finnois que du same. Le same et le finnois alternent également en dehors du milieu familial, aussi bien dans le cadre de l'école qu'en dehors - avec les voisins et les personnes inconnues - alors que la communication avec les employés ou fonctionnaires est plutôt caractérisée par l'usage presque exclusif du finnois. On dirait que la préservation du same pour les générations à venir n'est assurée qu'en partie, ce qui s'explique par le fait qu'une grande partie des parents de mariages mixtes utilisent en tant que langue de la famille aussi bien le same que le finnois, avec une prédominance, dans la plupart des cas, du finnois. 
Tableau $n^{\circ} 1$. Quelle langue parlez-vous avec les personnes mentionnées ici ?

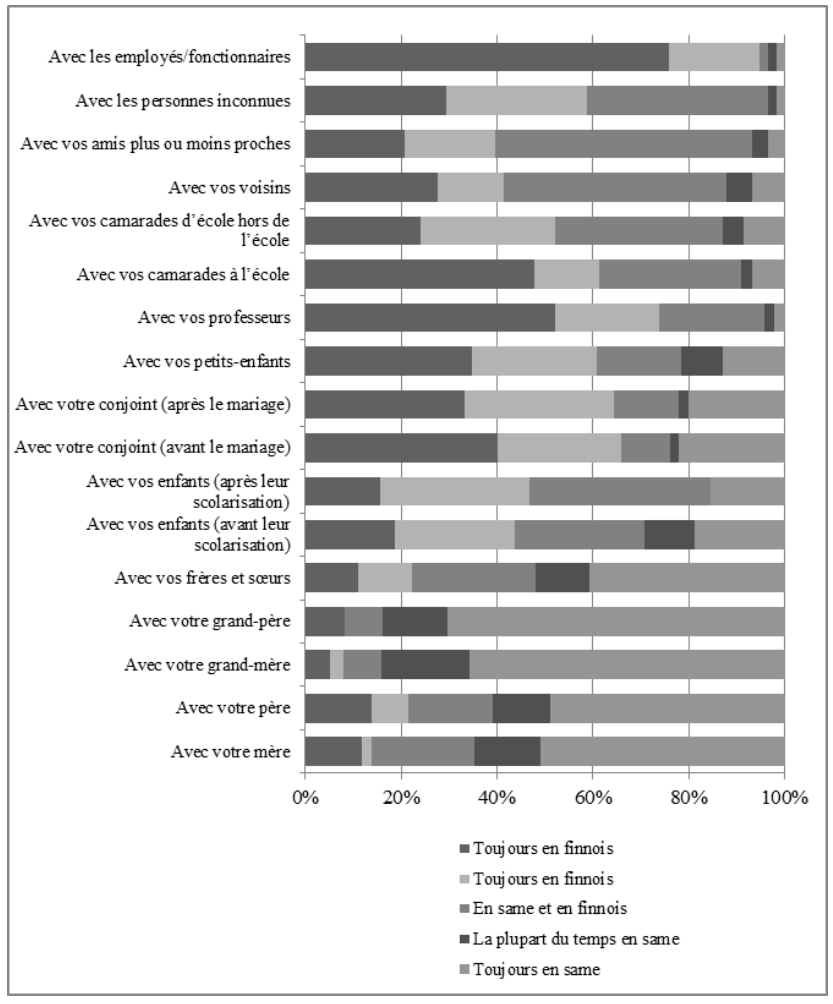

20 Il n'est pas inutile de revenir un moment sur le deuxième groupe de questions, qui est étroitement lié au premier, et où j'interroge les informateurs sur les préférences linguistiques de leurs interlocuteurs. Je cherchais à savoir s'il existe des interlocuteurs (surtout de deuxième ou de troisième génération) qui, afin de manifester leur attachement à leur langue ou encore afin de la transmettre, utilisent la langue same dans leur interaction avec mes informateurs. Les données recueillies de la sorte coïncident avec les résultats présentés dans le premier diagramme, ce qui nous permet d'établir que, selon toute probabilité, une interaction selon le modèle d'un choix de langue « mélangé » ne se présente pratiquement pas, c'est à dire que du point de vue du choix de la langue, les interactions des Sames avec leurs interlocuteurs sont caractérisées par la réciprocité, l'adaptation au choix de langue de l'interlocuteur.

Dans la troisième série de questions, nous trouvons des niveaux liés au quotidien des informateurs. La question posée portait sur la langue choisie par les informateurs dans les contextes formels. Le tableau ci-dessous résume les réponses à cette question et illustre le fait que les Sames parlent effectivement presque toujours finnois dans des contextes formels où il est peu probable que leur interlocuteur parle same. En revanche, dans les contextes où il leur est possible de parler same - comme à l'église, dans le cadre de l'élevage de rennes, de travaux communautaires ou de manifestations organisées par des Sames, le same et le finnois sont employés alternativement; dans le dernier cas, c'est en général l'emploi du same qui caractérise le choix de la langue. 
Tableau $^{\circ}{ }^{2}$ 2. Quelle langue utilisez-vous dans les situations suivantes?

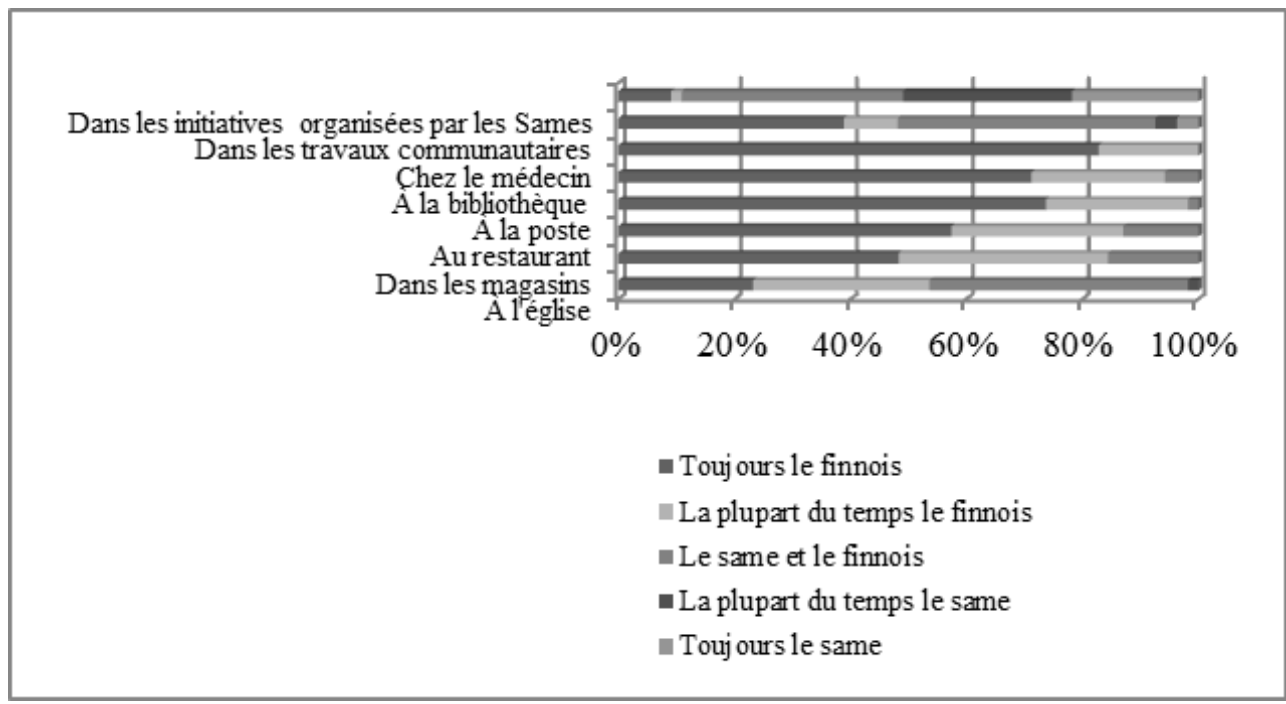

Cela fait plusieurs décennies que l'accès aux émissions de radio et de télévision et à la presse en same est assuré dans les territoires habités par les Sames; il paraît en Finlande de plus en plus d'œuvres littéraires signées par des Sames, de plus en plus de journaux et revues en same, et, à l'exception de quelques membres de la génération la plus âgée, tous les Sames sont alphabétisés. Compte tenu de ces éléments, je voulais avec la quatrième section du questionnaire savoir comment l'existence de médias influence les pratiques de choix linguistique, et jusqu'à quel point, dans cette communauté, la préservation des compétences en same à l'écrit va de pair avec la pratique des compétences de lecture et de compréhension. C'est là aussi que, grâce aux questions sur le calcul mental et sur les jurons, je cherche à savoir jusqu'à quel point la langue maternelle reste prédominante dans les automatismes.

\section{Tableau $\mathrm{n}^{\circ} \mathrm{3}$}

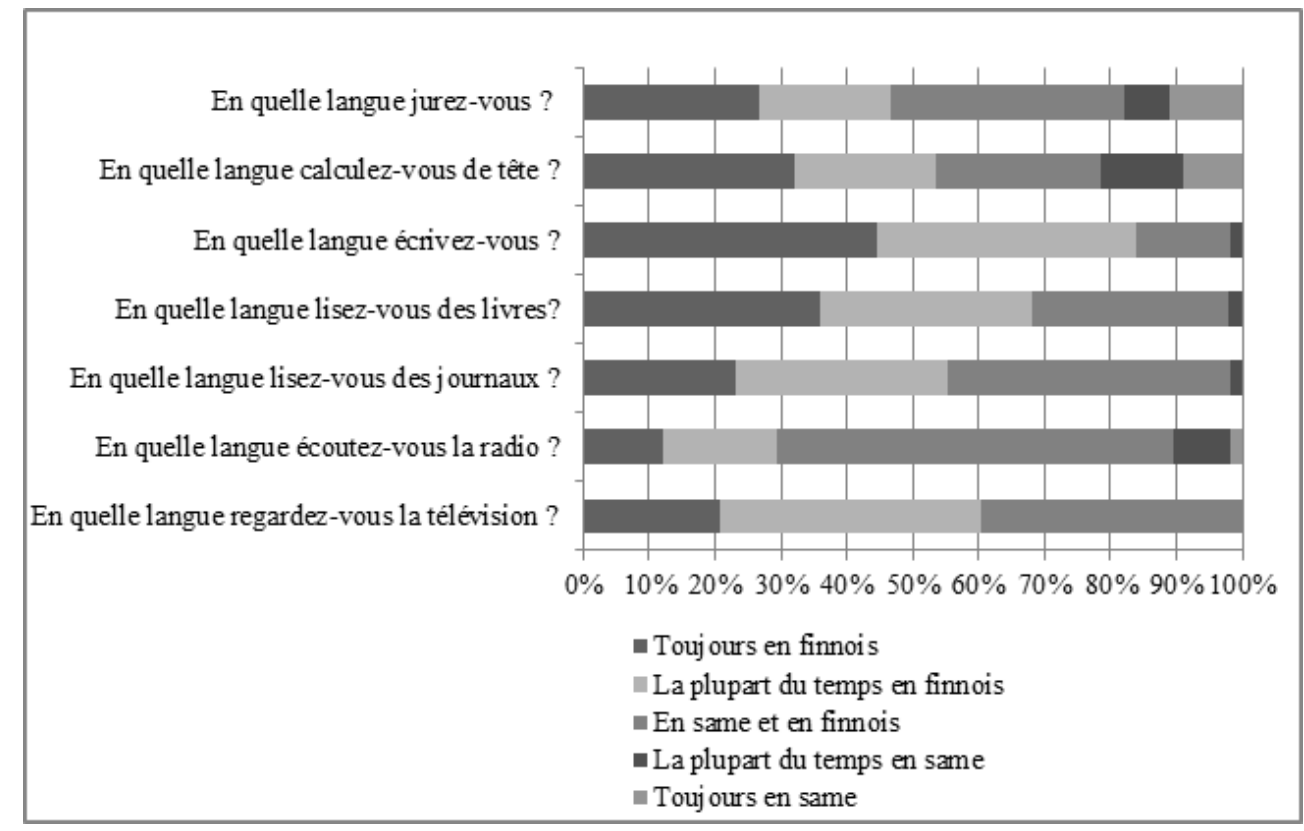



linguistiques peuvent être plus reliés à la langue maternelle que les capacités de lecture, d'écriture et de compréhension, c'est-à-dire qu'à cette étape du changement de langue le finnois majoritaire n'a pas entièrement remplacé la langue maternelle. De plus, le tableau qui se dessine montre que les membres de cette communauté maîtrisant de manière stable deux langues ou plus écrivent, lisent la presse et la littérature, et suivent des émissions de télévision aussi bien en finnois qu'en same. La prédominance du finnois pour ce qui est des compétences d'écriture saute aux yeux : plus de $80 \%$ des personnes interrogées écrivent en général ou exclusivement en finnois. Cela s'explique par les faiblesses de l'enseignement, et par les difficultés que les personnes les plus âgées ont encore aujourd'hui à écrire en same. Il faut souligner aussi que l'écoute de la radio touche davantage les Sames que les autres, car les émissions de radio en same ont une tradition plus longue que les émissions télévisées.

\section{Emploi informel de la langue et mutations sociales}

Comme nous examinons les modèles d'emploi de la langue de cette communauté bilingue conformément aux variables les plus fréquemment utilisées dans les recherches sur le changement de langue, à savoir en comparant l'âge, le sexe, la profession et la formation, nous sommes en mesure d'obtenir un tableau suffisamment nuancé de la situation linguistique de la communauté. Compte tenu de l'aménagement social et linguistique détaillé ci-dessus dans les communautés que j'ai étudiées et les transformations qui en découlent, on peut montrer clairement les mutations pour chacun des groupes d'âge. D'après nos observations antérieures, il peut y avoir des différences considérables dans les pratiques de choix de la langue entre les hommes et les femmes. Les recherches menées dans ce domaine sont contradictoires : tantôt les femmes sont plus disposées à préserver leur langue maternelle, tantôt elles sont majoritairement caractérisées par le choix de la nouvelle langue. Je me suis donc demandé ce qu'il en est dans ces communautés.

Les pratiques linguistiques révèlent également des différences suivant le niveau d'éducation et la profession. De ces points de vue, les différences que nous pouvons identifier sont des facteurs ambivalents dans le changement de langue (cf. Kloss 1967) : elles peuvent influer de manière positive ou négative selon les caractéristiques de la communauté. Dans de nombreux groupes, ceux qui ont un faible niveau de scolarisation ont mieux préservé les pratiques d'emploi de la langue que les autres. Dans la communauté considérée, nous pourrions attendre que dans les activités sames traditionnelles, l'élevage du renne ou l'artisanat, chez les personnes sans formation ou avec un bas niveau d'éducation et en contact surtout avec d'autres Sames, la langue soit mieux préservée que par les membres les mieux formés de la communauté same dont le réseau relationnel se compose surtout de Finnois.

J'ai suivi la méthode binaire traditionnelle, la prise en compte de plus de deux variables n'étant pas pertinente pour cette recherche.

La première section du questionnaire reflétait les pratiques linguistiques des informateurs dans le domaine informel, familial, que j'ai corrélées avec les quatre variables sociales. Puisque nous sommes partis de l'idée que les communautés étudiées sont plutôt caractérisées par le changement de langue que par le maintien de leur langue, on pourrait s'attendre à ce que les modèles de choix de langue se répartissent 
dans les quatre groupes de telle sorte que l'usage de la langue maternelle domine avant tout dans le cas des personnes les plus âgées, alors que chez les jeunes la langue prédominante serait le finnois. Le tableau $n^{\circ} 4$ montre bien que dans les situations de conversation informelle, les personnes interrogées utilisent la plupart du temps - à quelques exceptions près - les deux langues avec leurs interlocuteurs.

Tableau $n^{\circ} 4$. L'usage informel de la langue en corrélation avec l'âge

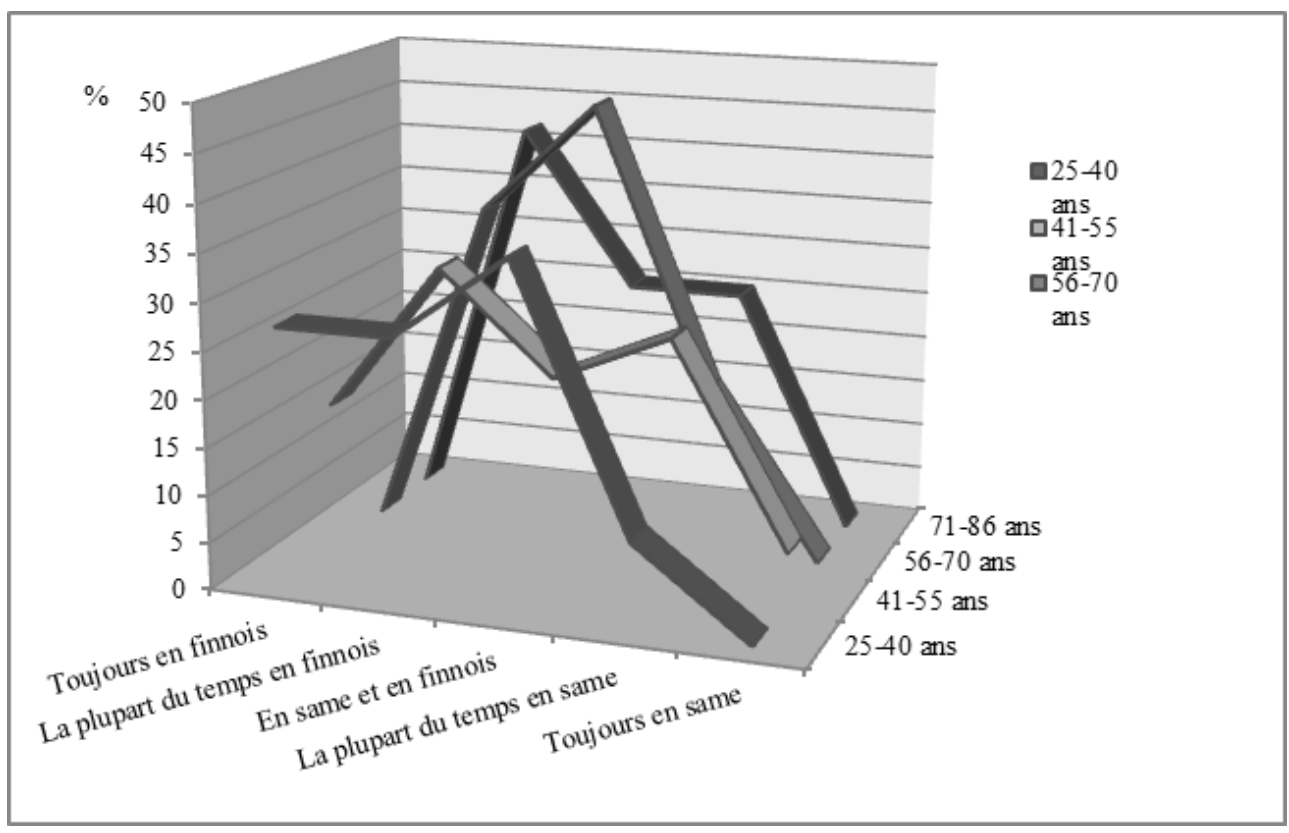

Chez les plus jeunes, on distingue effectivement une tendance à l'usage du finnois : $54 \%$ d'entre eux utilisent généralement ou exclusivement le finnois dans l'interaction informelle. Il est prometteur que 36 \% d'entre eux utilisent les deux langues, grâce surtout au développement de l'instruction en same et au fonctionnement des écoles en same. En tout, 9 \% d'entre eux préfèrent de manière générale le same.

En ce qui concerne la génération d'âge moyen avancé, personne ne choisit exclusivement l'une ou l'autre langue. Les réponses de la génération des 41-55 ans connaissent la répartition la plus équilibrée. On voit que les membres des deux groupes les plus âgés ont noté pour beaucoup qu'ils utilisent en général le finnois dans ce type de situations. Ceci est contraire à mes attentes et s'explique sans doute par le fait qu'en eux vit toujours l'expérience négative de voir leur langue longtemps reléguée à l'arrière-plan, alors que les jeunes et les personnes d'âge moyen ont grandi dans un milieu bilingue, où ils n'ont pas honte de parler same et où dans le cadre de la préservation de l'identité same, le maintien de la langue est devenu prioritaire. 
Tableau $n^{\circ} 5$. Corrélations entre l'emploi de la langue et le sexe

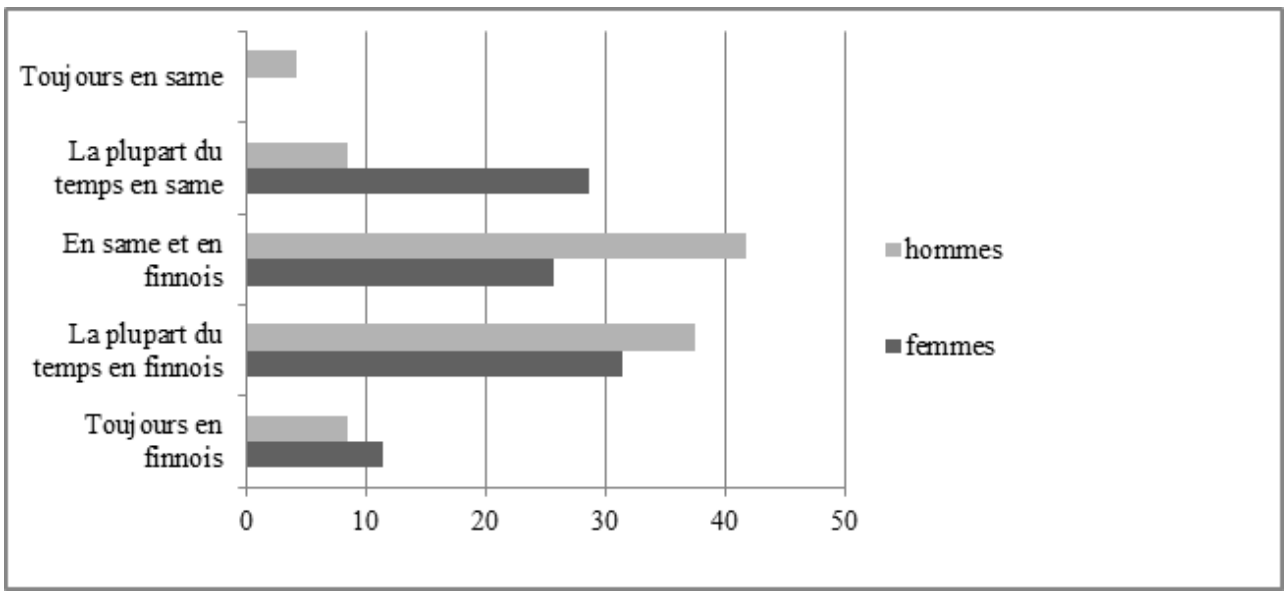

Pour l'usage de la langue par les hommes et par les femmes, je m'attendais à ce que les femmes préservent mieux leur langue que les hommes dans le cercle familial. Le tableau 5 ne confirme qu'en partie cette attente. Si en situation informelle les hommes font en général appel aux deux langues ( $42 \%$ ) ou principalement au finnois $(37,5 \%)$, la même proportion de femmes que d'hommes (31\%) utilise principalement le finnois, mais la proportion de femmes utilisant principalement le same est beaucoup plus élevée (29\%) que celle des hommes (8\%).

Cette distribution m'a incitée à étudier quels autres facteurs peuvent influer sur les choix linguistiques des femmes. L'utilisation des deux langues à part à peu près égale s'explique entre autres par les pratiques linguistiques différentes des femmes dans le cadre d'un mariage endogame ou exogame. Dans un ménage same, la femme choisit, bien sûr, de parler same dans un contexte informel. Dans un mariage mixte samefinnois et dans un contexte informel multilingue, l'usage linguistique est caractérisé par l'usage à égalité du finnois et du same, ou encore du finnois de manière prédominante ou exclusive.

Ma supposition était que du point de vue des rapports entre profession et usage de la langue, bien que les éleveurs de rennes préservent leur langue mieux que les gens qui ont d'autres emplois, leurs interactions informelles et familiales seraient de plus en plus déterminées par la prédominance du finnois, puisque le choix linguistique des informateurs, comme nous l'avons vu ci-dessus, est avant tout déterminé par la langue de leur entourage le plus proche. 
Tableau $n^{\circ} 6$. Corrélations entre l'emploi de la langue et la profession

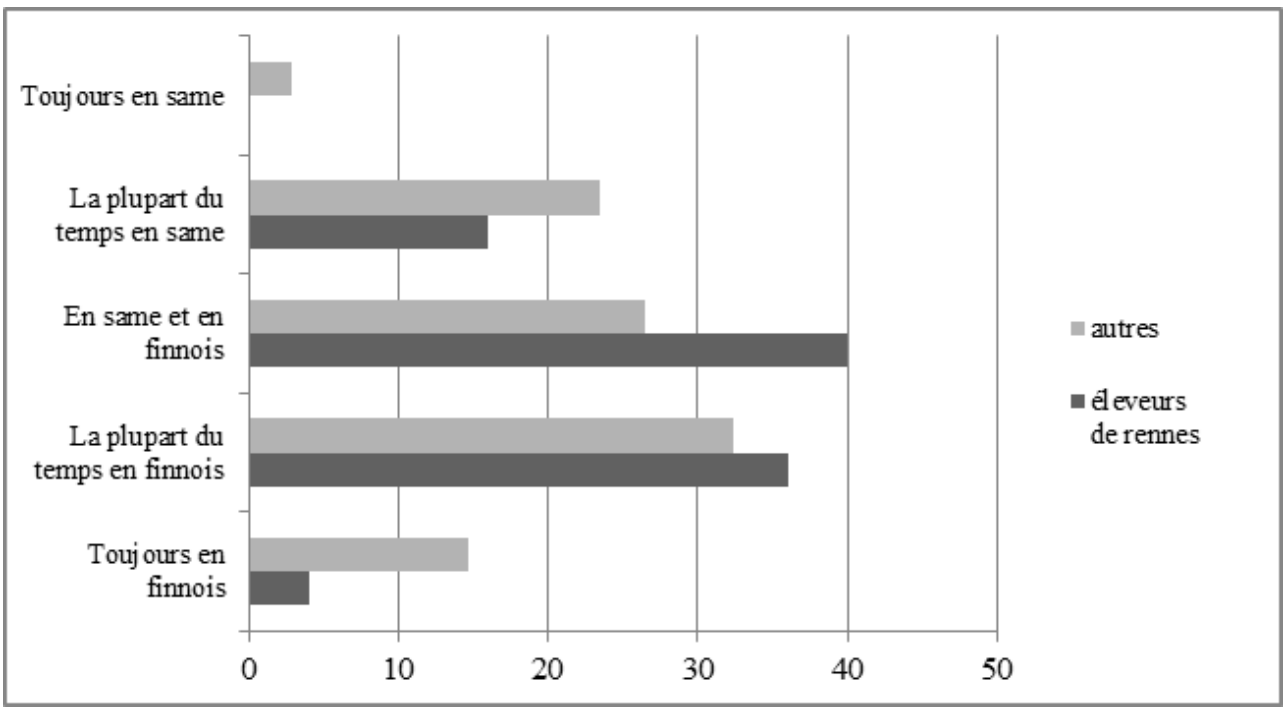

Dans le diagramme ci-dessus, de manière non surprenante, nous voyons qu'une grande proportion d'éleveurs de rennes ( $40 \%)$ utilisent les deux langues, plus que les autres $(26 \%)$. On peut donc estimer que l'existence d'une terminologie same de l'élevage des rennes et les travaux collectifs rassemblant des locuteurs du same jouent un rôle de maintien de la langue.

Le glissement vers le finnois de la langue utilisée par les éleveurs de rennes n'est pas étonnant si nous pensons à l'influence des différents facteurs sur le choix de la langue. Il semble évident que la langue prédominante pour les éleveurs de rennes vivant en situation de mariage mixte est le finnois. Une autre raison de ce glissement est que les communautés d'éleveurs de rennes s'étant diluées avec l'arrivée de nombreux locuteurs du finnois, dans la plupart des cas la communication s'y fait également en finnois - à l'exception des activités liées aux travaux collectifs. La moitié de ceux qui exercent d'autres professions parlent aussi, en général ou exclusivement, finnois. Il faut noter cependant que $27 \%$ d'entre eux parlent en général ou exclusivement same, alors que parmi les éleveurs de rennes personne ne mentionne l'usage exclusif du same. Parmi ceux qui exercent d'autres professions, on trouve les quelques retraités qui, malgré leur âge avancé, élèvent des rennes ou ont d'autres activités traditionnelles sames. La plupart des plus âgés vivent en mariage endogame. L'examen détaillé des réponses révèle que ces personnes âgées, ces ménages sames se livrant à des activités traditionnelles sames, couvrent les $3 \%$ parlant exclusivement same. À peu près la moitié des autres personnes ayant des professions différentes ne peuvent pas être rattachées au mode de vie traditionnel same, et les données montrent que celles-ci choisissent en général le finnois comme langue d'interaction informelle.

Le tableau $\mathrm{n}^{\circ} 7$ montre la corrélation entre le niveau de formation et l'usage de la langue. En Finlande, la tendance est à ce que beaucoup des Sames jouissant d'un haut niveau de formation quittent leur terre natale et aillent s'installer dans les territoires méridionaux ou dans les grandes villes de Laponie en quête d'un meilleur statut socioéconomique. Dans la mesure où la moitié des membres de la communauté que j'ai examinée ayant fait des études secondaires ou supérieures n'ont pas quitté le milieu familial, ou bien sont retournés s'installer en Laponie, je m'attendais à ce qu'ils utilisent 
leur langue autant que ceux qui n'ont pas de formation, c'est à dire à ce que la corrélation entre niveau de formation et usage de la langue soit minime.

Tableau $n^{\circ} 7$. Corrélations entre l'emploi de la langue et le niveau d'études

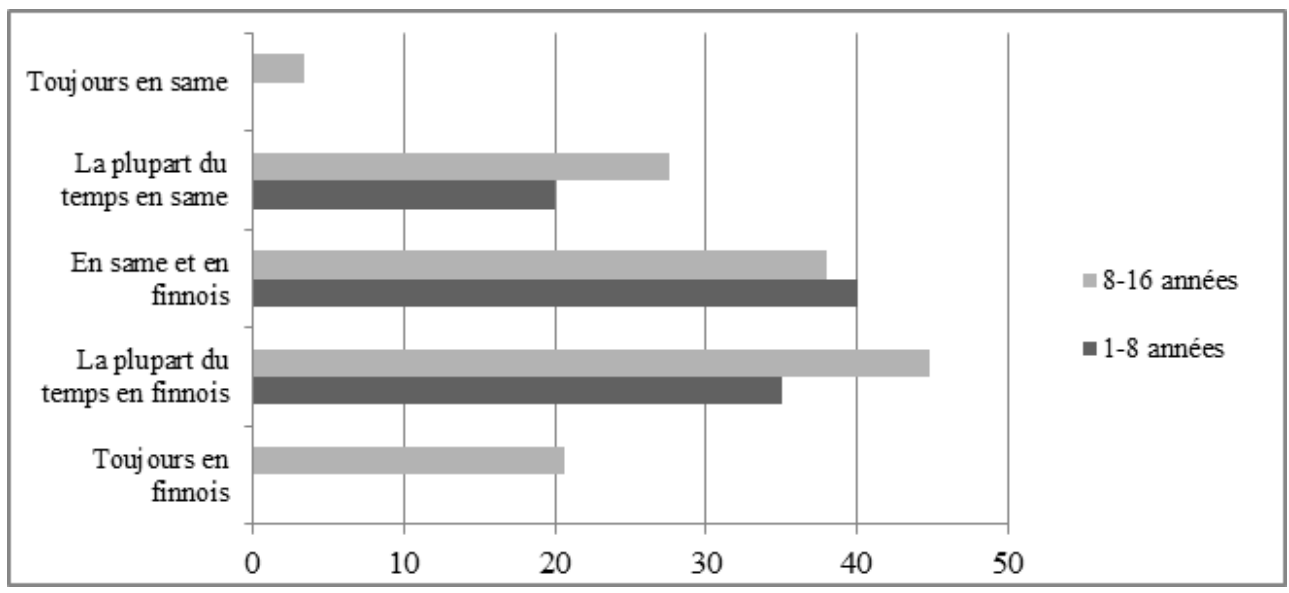

Le diagramme montre clairement que bien qu'en milieu informel les personnes les mieux formées utilisent le plus souvent le finnois (66\%), elles sont pourtant nombreuses $(30 \%)$ à utiliser généralement ou exclusivement le same. On les trouve dans les jeunes générations : une partie vit en mariage endogame, mais la plupart sont restées dans leur milieu familial samophone et entretiennent avec celui-ci une communication quotidienne. Certaines sont des enseignants de same, pour qui la préservation et la transmission de la langue sont particulièrement importantes. Les réponses des informateurs les mieux formés se partagent entre l'emploi alterné des deux langues et l'usage général de telle ou telle langue. La seule différence entre les personnes les mieux formées et celles moins formées est que personne, parmi ces dernières, n'utilise une seule langue exclusivement.

En résumé, on peut dire qu'aucun des facteurs sociaux n'influe clairement sur le choix de la langue, c'est-à-dire que les normes qui régissent ce dernier pour les Sames en milieu informel ne dépendent que dans une faible mesure de l'âge et du sexe des informateurs, et presque aucunement de leur profession et de leur niveau d'éducation. Une analyse plus approfondie des tableaux ci-dessus introduit cependant des nuances et témoigne de la légère prédominance du finnois et en tout cas d'un équilibre dans le bilinguisme. La situation de changement de langue pourrait être décrite avec plus de précision si on pouvait examiner non seulement l'influence mutuelle de deux variables, mais faire intervenir plusieurs variables, ce qui montrerait le caractère lâche ou étroit des corrélations.

\section{Usage formel de la langue et corrélation avec les variables sociales}

J'examine ci-dessous les corrélations entre des facteurs comme l'âge, le sexe, la profession et le niveau de formation d'une part et le choix de la langue par les informateurs dans les contextes formels, par exemple à l'église, dans les magasins, chez le médecin. Les résultats s'appuient sur les réponses à la troisième section du questionnaire. 


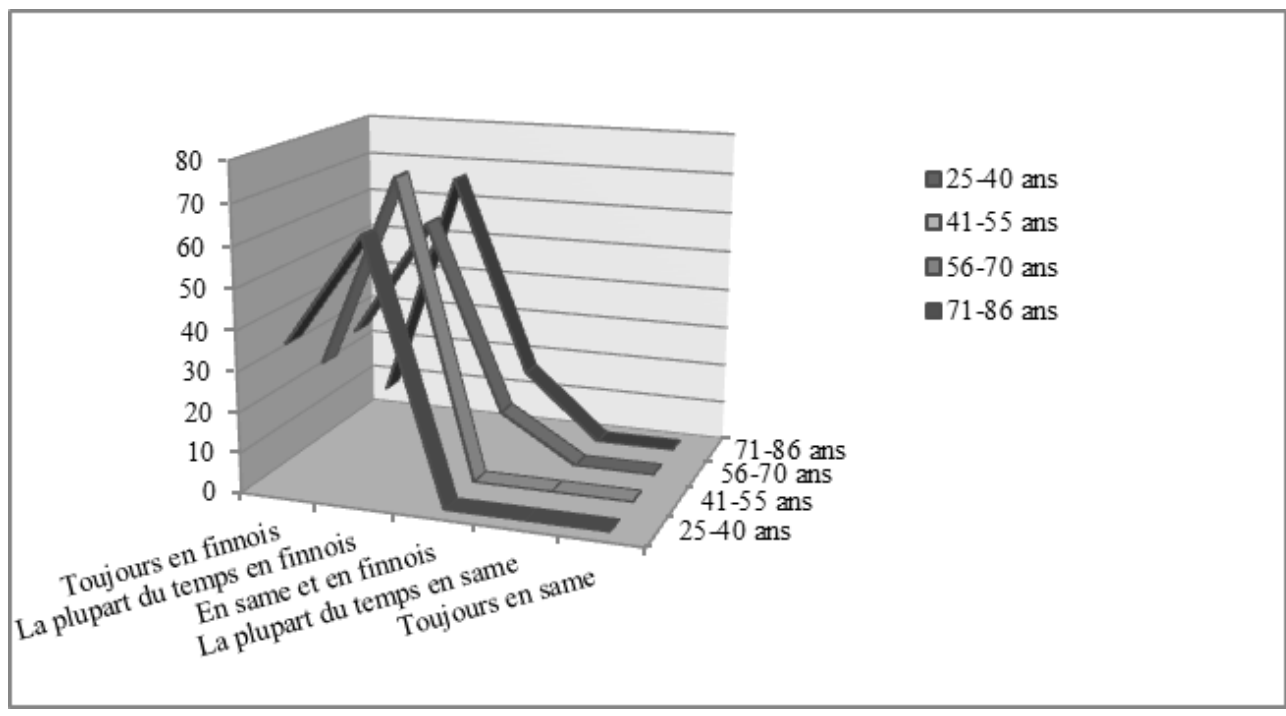

Les moyennes présentées dans le tableau $n^{\circ} 8$ semblent démontrer que la caractéristique principale du rapport entre l'âge et l'usage formel de la langue est que seule la classe d'âge la plus élevée utilise en situation formelle aussi bien le same que le finnois, alors que les plus jeunes n'utilisent aucunement le same, autrement dit leur choix tombe soit exclusivement soit généralement sur le finnois. Mais l'examen attentif des résultats montre que la moyenne occulte le fait non insignifiant que dans les manifestations organisées pour les Sames $21 \%$ parlent same et finnois, $16 \%$ généralement same et $12 \%$ exclusivement same. Pour ce qui est des classes d'âge, ce qui se dessine est que dans les jeunes générations, le same y est parlé soit généralement, soit exclusivement : $45 \%$ d'entre eux parlent toujours same, ce qui est réjouissant du point de vue du maintien de la langue. La question se pose logiquement de savoir quels sont les cadres formels où les personnes plus âgées, à la différence des jeunes, emploient les deux langues. Un examen attentif des données montre clairement qu'il s'agit d'abord des manifestations sames, et en deuxième lieu de l'église, qui fait partie du quotidien des personnes âgées, ainsi que des travaux collectifs dans l'élevage de rennes.

Étant donné l'importance que revêtent les résultats partiels occultés par les moyennes pour la préservation du same, il a fallu réexaminer les méthodes d'analyse des questionnaires. Les difficultés d'analyse proviennent clairement du fait que les manifestations sames et, dans une moindre mesure, les travaux collectifs ne peuvent être considérés comme des cadres formels. Dans ces cadres plutôt semi-formels, les pratiques linguistiques diffèrent de façon significative de celles rencontrées dans les situations formelles. Le lien entre le choix de la langue dans les situations formelles et les trois autres variables a donné des résultats similaires, donc c'est la prédominance $\mathrm{du}$ finnois qui caractérise les usages linguistiques dans les situations formelles, indépendamment du sexe, de la profession et de la formation.

41 Les normes d'usage de la langue diffèrent pour les hommes et les femmes; dans les cadres formels dominés par des Finnois unilingues, les hommes choisissent en général le finnois comme langue d'interaction, tandis que les femmes utilisent le finnois et le same. De même, dans d'autres situations formelles, le choix linguistique des femmes est 
caractérisé par l'usage des deux langues. Les données occultées mettent également en évidence le rôle que jouent les femmes dans la protection de la langue maternelle, montrant que ce sont elles qui, dans les manifestations sames, s'expriment le plus souvent en same.

Par rapport à la profession, on constate que dans les cadres formels c'est le finnois qui prédomine, indépendamment de la profession. Les éleveurs de rennes choisissent généralement le finnois; dans la grande majorité des cas, les membres exerçant d'autres professions s'expriment toujours en finnois.

Tableau $n^{\circ}$ 9. Corrélations entre l'emploi formel de la langue et la formation

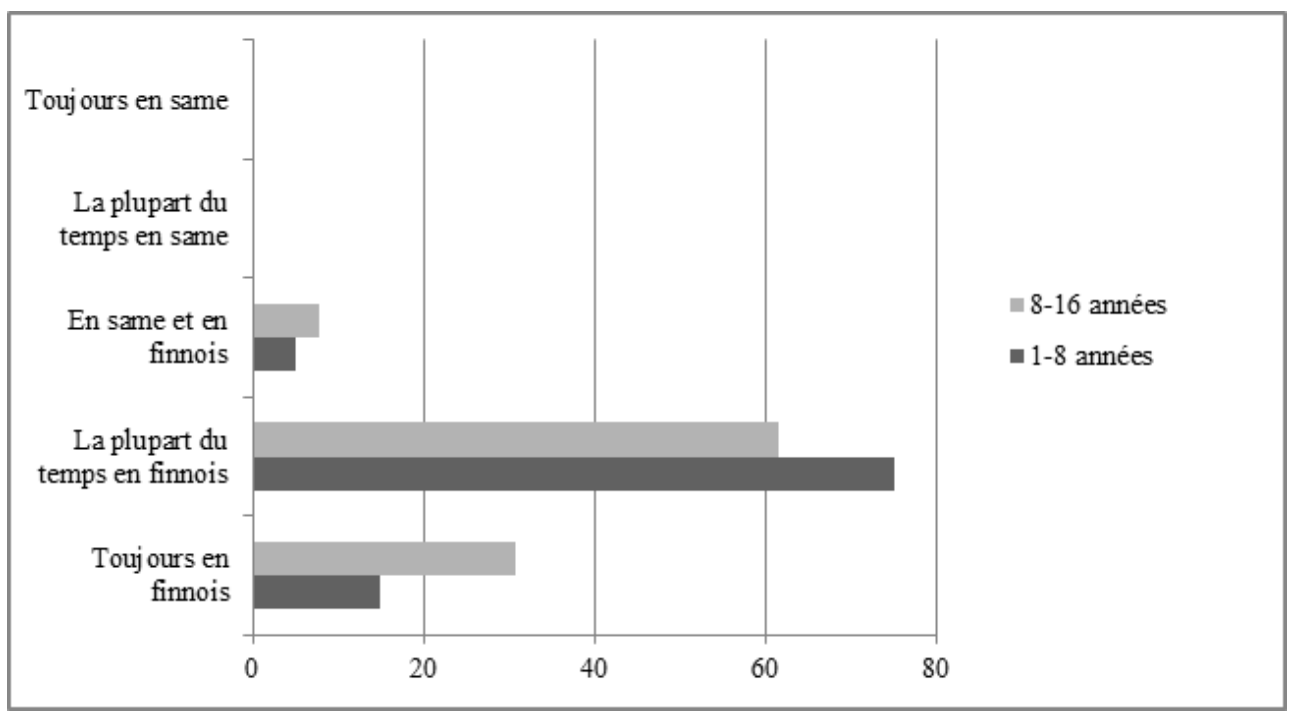

En ce qui concerne la corrélation entre le choix de la langue dans les situations formelles et le niveau de formation, étant donné le manque de représentation des situations semi-formelles mentionnées ci-dessus, nous pouvons dire que c'est toujours l'usage du finnois qui prédomine dans les deux groupes. J'ai réexaminé soigneusement les choix de langue des sames dans les manifestations organisées pour eux et dans les travaux collectifs. Il en est ressorti que dans les manifestations sames, en ce qui concerne les membres ayant fait des études avancées (61\%), c'est généralement ou exclusivement l'usage du finnois qui est choisi, alors que ceux ayant le niveau d'instruction le plus bas utilisent soit les deux langues soit le same dans des proportions différentes (90\%). Dans les travaux collectifs, cependant, l'emploi du same diminue dans les deux groupes; dans ce contexte semi-formel, le choix de l'alternance des deux langues ou celui du same caractérise plutôt les moins instruits (50\%); ceux qui ont fait des études avancées utilisent presque exclusivement le finnois (93\%).

En résumé, nous pouvons dire que dans les contextes formels c'est l'usage du finnois qui prédomine, indépendamment de l'âge, du sexe, de la profession et de la formation. Cependant, l'une des raisons d'être des manifestations sames est la préservation de la langue. Elles créent des occasions pour parler le same et concernent essentiellement les jeunes générations, les personnes les moins instruites et les femmes. De même, ce sont les femmes qui ont le plus tendance à pratiquer le same en plus du finnois dans les cadres formels, donc il semble que ce soient elles qui jouent le plus grand rôle dans la préservation de la langue. C'est la pratique alternée du same et du finnois qui caractérise les choix linguistiques des plus âgés. Cette pratique est rendue possible, en 
premier lieu, grâce au maintien du same à l'église et ensuite grâce aux manifestations communautaires. Les activités traditionnelles comme l'élevage des rennes et l'artisanat semblent aussi contribuer, dans une moindre mesure, à la conservation de la pratique alternée des deux langues.

Il me semble que pour mieux comprendre la situation de changement de langue des Sames, il est également important d'étudier le rôle des médias dans la préservation de la langue dans les différentes classes d'âge et de voir si l'éducation a une influence sur le choix de la langue dans laquelle les informateurs lisent, écrivent ou suivent les émissions de télévision et de radio. Mon hypothèse est que le sexe et la profession n'ont pas une influence marquante sur le choix de la langue. Les résultats s'appuient sur la section IV du questionnaire.

La corrélation entre les réponses obtenues pour la question «En quelle langue suivezvous en général vos émissions de télévision? » et l'âge montre qu'il n'y a pas, à cet égard, de différence marquante entre les jeunes et les plus âgés. $45 \%$ des plus jeunes, $31 \%$ de la classe intermédiaire, $47 \%$ des plus âgés et $33 \%$ des très âgés suivent des émissions de télévision dans les deux langues. Naturellement, qu'une proportion aussi élevée de jeunes suivent les émissions de télévision également en same, est, de toute façon, un fait très positif. Il est cependant intéressant de voir que $36 \%$ des plus jeunes ne regardent que des émissions en finnois, alors qu'en ce qui concerne les autres générations, seulement $10 \%$ choisissent exclusivement le finnois, ce qui s'explique par le fait que la proportion du nombre des programmes en same pour les petits est très faible. Les données montrent également que ce sont les hommes sames éleveurs de rennes qui passent le plus de temps à regarder des émissions en same et en finnois, probablement parce qu'une grande partie des documentaires et des reportages en same a trait à la conservation du mode de vie traditionnel des Sames et concerne ainsi les droits des éleveurs de rennes et leur situation présente et future. Du point de vue des émissions de télévisions, le rapport entre le niveau de formation et le choix de la langue est non significatif. Dans ce cas, l'accessibilité, la quantité et la qualité des émissions en same expliquent beaucoup mieux les choix de langue.

47 Le fait que les émissions de radio en same soient plus nombreuses que les émissions de télévision et que leur diffusion recouvre toute la Laponie finlandaise explique de façon incontestable pourquoi les quatre générations d'âge privilégient dans une aussi grande proportion les émissions de radio en same ou en finnois ( $58 \%$; $70 \%$; $57 \%$; $45 \%) .20 \%$ de la population âgée et $15 \%$ de la population d'âge moyen avancé écoutent en général ou exclusivement des émissions de radio en finnois. Les données montrent également que les femmes qui ne s'occupent pas de l'élevage de rennes écoutent un peu plus d'émissions en same que les hommes éleveurs de rennes, qui passent la plus grande partie de la journée à l'extérieur de la maison. Le rapport entre le niveau d'éducation et l'écoute des émissions de radio en same est moins explicite.

Une très grande partie de la génération la plus âgée, $66 \%$, lit en général ou exclusivement des journaux écrits en finnois et $25 \%$ les lisent dans les deux langues indifféremment. Le groupe d'âge moyen avancé et celui des jeunes les plus âgés les lisent dans les deux langues. Cette différence peut venir du fait que les journaux en same - ou plus précisément les revues - sont difficiles à se procurer pour les personnes âgées vivant dans les communautés les plus isolées. Les données révèlent également que pour ce qui est de la lecture dans la langue maternelle, le facteur du niveau d'études est plus important que celui du sexe ou de la profession des informateurs. 
Parmi les moins instruits, personne ne lit en général ou exclusivement les journaux en same, en revanche $70 \%$ d'entre eux lisent en général ou toujours en finnois. $60 \%$ des plus instruits, qui lisent couramment aussi bien le same que le finnois, lisent les journaux dans les deux langues, et $3 \%$ les lisent généralement en same.

Pour la lecture des livres, les usages de la génération la plus âgée diffèrent légèrement de ceux de la lecture des journaux. D’après les données, ils lisent plus de livres écrits en same que les plus jeunes. On voit tout de suite que plus de la moitié de la génération des 41-55 ans lit des livres exclusivement en finnois, alors que, dans la même proportion, les plus jeunes lisent "en général en finnois ", c'est à dire en same et finnois indifféremment. Manifestement, les éleveurs de rennes préfèrent les livres écrits en same, car seulement $12 \%$ d'entre eux lisent exclusivement en finnois, alors que plus de la moitié des membres exerçant d'autres professions ne lit que des livres en finnois. Il n'y a pas de rapport entre le sexe et les habitudes de lecture des livres. On ne décèle pas non plus de différences essentielles en ce qui concerne le niveau d'études, à part le fait que contrairement à ce qui se passe pour la lecture des journaux, ce qui caractérise les plus instruits, c'est que $64 \%$ d'entre eux préfèrent, dans une plus ou moins grande mesure, lire des livres en finnois, contre $40 \%$ pour les moins instruits. $10 \%$ d'entre ces derniers lisent en général des livres en same, tandis que parmi les plus instruits personne ne lit exclusivement ou généralement en same.

Comme je l'ai mentionné plus haut lors de l'analyse des réponses aux questionnaires sur les usages linguistiques, sur le plan de la lecture c'est le same qui prédomine ; en revanche, pour l'écriture, en raison de la faiblesse des compétences, c'est l'écriture du finnois qui est la plus répandue dans les communautés sames. Pour ce qui est de l'écriture, il a des différences sur lesquelles il faut s'arrêter, tant en fonction de l'âge que du niveau d'éducation. La plus jeune génération - qui a aujourd'hui la possibilité de bénéficier d'une éducation bilingue efficace et qui peut ainsi acquérir des compétences linguistiques - écrit autant en same qu'en finnois (27\%); c'est la seule des quatre générations qui écrit le plus souvent dans les deux langues, à peine plus de $10 \%$ des membres des trois autres générations écrivent dans les deux langues. $91,5 \%$ des hommes écrivent en général ou toujours en finnois, alors que chez les femmes la proportion n'est que de $71 \%$. Les femmes sont nettement plus nombreuses à écrire dans les deux langues ( $20 \%$ ) que les hommes ( $4 \%$ ), et c'est chez les femmes également que $3 \%$ écrivent en général en same : aucun des hommes interrogés n'écrit en général ou exclusivement en same. On pouvait se douter que plus quelqu'un serait éduqué plus il écrirait en same et en finnois indifféremment, et également que la formation n'aurait pas vraiment d'incidence sur le fait que quelqu'un écrive généralement ou exclusivement en finnois; dans les deux cas, c'est la préférence du finnois qui caractérise le choix, indépendamment du niveau d'instruction.

Dans l'ensemble, on peut dire qu'en ce qui concerne les médias, les usages linguistiques ne diffèrent pas de façon significative selon l'âge, le sexe, la profession et la formation, excepté dans les cas suivants: (i) dans le cas des émissions de radio, le choix des émissions en same est plus fréquent, car comparées aux émissions de télévision en finnois elles sont plus aisées à capter dans toutes les communautés sames des territoires étudiés; (ii) les plus âgés sont plus nombreux à lire des livres en same que les plus jeunes, pour la lecture des journaux en same c'est exactement l'inverse ; (iii) les compétences d'écriture dans la langue maternelle sont supérieures chez les jeunes du fait qu'ils peuvent bénéficier de l'enseignement bilingue; (iv) les études renforcent 
l'aptitude à écrire dans la langue maternelle, ceux qui ont un niveau d'études avancé et les femmes écrivent plus fréquemment en same. Ces remarques font ressortir le fait que la communauté étudiée accède aux productions des médias et aux œuvres littéraires en same presque indépendamment de l'âge, du sexe, de la profession et de la formation - bien que dans une proportion limitée - et les accueille même favorablement. Dans l'intérêt du maintien durable de la langue, faire progresser ces chiffres et ces possibilités doit être une tâche prioritaire, qui viendrait soutenir d'une façon active les compétences de lecture et d'écriture des plus jeunes et développerait leur goût à lire et à écrire dans la langue maternelle.

\section{Conclusion}

En tenant compte des présupposés et des résultats, ce qui caractérise surtout la situation actuelle de changement de langue des communautés linguistiques d'Enontekiö et de Sodankylä, c'est que (i) l'emploi exclusif ou habituel de la langue same se trouve relégué en premier lieu aux situations familiales, ensuite aux autres niveaux informels d'emploi de la langue (interaction avec les voisins, les amis, les camarades de classe, à l'église, lors des travaux collectifs et dans les manifestations organisées pour les Sames); (ii) dans leurs interactions avec les générations plus jeunes, les informateurs, pour communiquer dans les situations formelles choisissent plus souvent le finnois majoritaire que le same; (iii) les samophones des mariages mixtes emploient de moins en moins leur langue maternelle; (iv) c'est l'usage du finnois majoritaire qui prédomine chez les enfants issus de mariages mixtes; (v) les variables personnelles, c'est-à-dire l'âge des locuteurs, leur sexe ou leur profession n'ont qu'une incidence minime sur les normes du choix de la langue dans les niveaux formels et informels.

53 Tous ces facteurs pourraient contribuer à l'accélération du changement de langue, s'il n'y en avait pas de nombreux autres venant contrecarrer le recul progressif de la langue minoritaire, tels que, par exemple, l'enseignement de plus en plus populaire de la langue maternelle et les institutions, subventionnées par l'État, qui s'occupent de façon de plus en plus intense du développement de la langue, de la protection des intérêts des Sames, et qui soutiennent les activités favorisant la préservation de la langue maternelle liées au mode de vie traditionnel, tout spécialement l'élevage de rennes et l'artisanat, ainsi que les manifestations organisées pour les Sames, qui représentent une partie importante de la culture same. Pour ce qui est de la préservation de la langue, j'attribue un rôle prépondérant aux attitudes incontestablement positives concernant la langue maternelle et le bilinguisme samefinnois, que j'ai étudiés en détail dans ma thèse de doctorat. Les résultats obtenus montraient que la communauté étudiée, pratiquement sans exception, considérait comme extrêmement importants le maintien et la transmission de la langue maternelle et son appropriation par les jeunes générations, qu'elle ne trouvait pas la situation de bilinguisme same-finnois contraignante, qu'elle était étroitement attachée à la communauté de langue maternelle et à la valeur de la langue maternelle, même si elle n'était plus complètement persuadée de son utilité.

54 Mon hypothèse est donc que les attitudes positives rencontrées au cours de mon étude pourraient de plus en plus se retourner contre le finnois dominant, freinant le processus de changement de langue; elles pourraient, avec de la chance, promouvoir 
l'usage de la langue maternelle, surtout dans la vie des plus jeunes générations. Un autre fait encourageant est que c'est justement ces générations-là qui ont des attitudes résolument positives concernant leur langue maternelle. La langue maternelle reste encore aujourd'hui, pour la plupart des jeunes, un élément d'importance capitale pour leur identité, malgré le fait qu'ils choisissent de moins en moins le same comme moyen d'interaction dans différentes situations linguistiques. De même que l'attitude, l'identité linguistique des Sames a beaucoup changé dans les dernières décennies. Dans la communauté same d'aujourd'hui, on peut constater que toutes les générations s'éloignent progressivement de l'identité linguistique basée sur l'usage linguistique de la famille pour aller vers une identité communautaire modelée par un environnement linguistique élargi (c'est-à-dire une situation de bilinguisme dans laquelle la deuxième langue est dominante), les traditions culturelles des Sames, l'intégration de celles-ci dans la vie quotidienne et les attitudes très positives concernant la langue maternelle. Il ne faut pas, cependant, oublier que, quelle que soit l'ampleur de l'adhésion de la minorité au maintien de sa langue maternelle et quels qu'équilibrés soient les rapports entre la minorité et la majorité, cela ne garantit pas pour autant la survie de la langue. Le gage de la réussite de la conservation de la langue serait de toute façon le maintien de la langue maternelle au sein de la famille, sans quoi le processus de changement de langue peut tout au plus être ralenti, mais non stoppé. C'est pourquoi il est primordial de développer le prestige de la langue en portant les efforts sur la revitalisation de l'usage de la langue et sur la politique de l'enseignement des langues.

Que ma recherche, dont le but initial était de dresser un tableau de la situation de changement de langue dans les communautés sames d'Enontekiö et de Sodankylä, ait été menée à bien ne signifie pas pour autant qu'elle soit terminée. Les usages linguistiques actuels - et aussi, naturellement, les attitudes linguistiques - changent avec le temps. C'est pourquoi cette évaluation de la situation sera suivie par un examen des changements intervenus, qui utilisera les mêmes procédés méthodologiques et garantira la possibilité de comparaison des données. ASP Erkki, 1965, Lappalaiset ja lappalaisuus, Turku, Annales Universitatis Turkuensis. C2. ASP Erkki, 1966, The Finnicization of the Lapps, Turku, Annales Universitatis Turkuensis. B 100. DURAY Zsuzsa, 2008, Kétnyelvüség és nyelvi attitüd finnországi északi számi beszélóközösségekben, doktori disszertáció. (unpublished) FISHMAN Joshua, 1968, Readings in the Sociology of Language, The Hague, Paris, Mouton. GAL Susan, 1979, Language Shift: Social Determinants of Linguistic Change in Bilingual Austria, New York, San Francisco, London, Academic Press. HELANDER Elina, 1984, Om trespråkighet: en undersökning av språkvalet hos samerna i Övre Soppero, Umeå, Universitetet i Umeå. KLoss Heinz, 1967, Types of Multilingual Communities: A Discussion of Ten Variables, in FISHMAN Joshua, Language Loyality in the United States, The Hague, Mouton, pp. 206-252. MAGGA Tuomas, 1988, Saamelaisten koulutuskysymys Suomessa, Ruotsissa ja Norjassa. Kasvatustieteen sivulaudaturtyö, Oulu, Oulun yliopiston kasvatustieteiden tiedekunta. MÜLLER-WILLE Ludger, 1996, Kahden kultturin kohtaaminen. Saamelaiset ja suomalaiset Utsjoella, [1974. Lappen und Finnen in Utsjoki (Ohcejohka), Finnland. Münster.] Arctic Centre Reports 18, Rovaniemi, Lapin Yliopisto, Arktinen keskus. 
NICKUL Karl, 1970, Saamelaiset kansana ja kansalaisina, Helsinki, SKST 297.

SAMMALlaHTI Pekka, 1998, The Saami Languages. An Introduction, Karasjok, Davvi Girji OS.

SIURUAINEN Eino, 1977, Väestö- ja kielisuhteet sekä saamelaisuusaste. Suomen saamelaisalueen väestöstä ja sen toimeentulosta, Oulu, Oulun Yliopisto. Pohjois-Suomen Tutkimuslaitos.

WEINREICH Ulrich, 1953, Languages in Contact: Findings and Problems, New York, Linguistic Circle.

\section{NOTES}

2. Parmi les travaux présentant la situation antérieure des Sames du point de vue linguistique sous tel ou tel aspect, soulignons ceux qui permettent de mieux comprendre le processus de changement de langue. Telle est la première étude à caractère démographique, Nickul 1970, qui a servi de point d'appui pour déterminer les Sames habilités à voter. Asp a enrichi la littérature traitant des Sames dans deux travaux sociologiques $(1965,1966)$, dont le premier présente la situation sociale et économique à son époque, alors que le deuxième aborde la question de la perte d'identité et le processus d'assimilation culturelle unilatérale, la finnisation. L'éveil national des années 1970 a conduit à la revitalisation des études sames, surtout dans le domaine des droits des peuples minoritaires. Müller-Wille (1996) estime que cette revitalisation n'était qu'une réaction d'autodéfense tournée contre la pression extérieure de l'État et sa politique d'assimilation. À cette époque, l'Académie finlandaise des sciences subventionna de nombreuses études destinées à cartographier la société same et la situation socio-économique des Sames. Siuruainen (1977), dans son travail, présente la société same des années 1970. Ce sont surtout des chercheurs étrangers qui ont étudié le rapport entre majorité et minorité, et la question des droits des Sames à la fin des années 1970 (cf. Müller-Wille 1996). En Finlande, les Sames euxmêmes, dans le domaine des sciences sociales, ont pris surtout l'initiative de travaux linguistiques. Les années 1980 ont vu paraître des thèses de doctorat sur les Sames (dont les travaux d'Elina Helander en 1984 et de Tuomas Magga en 1988). Ces recherches sociolinguistiques fondées sur la coopération de chercheurs finnois et sames témoignent déjà de méthodes de terrain différentes. De plus en plus, des étudiants sames venant de terminer leurs études furent associés aux travaux de terrain, et ils purent par la suite participer à des projets de recherche plus importants. Ces études avaient été lancées, dans un premier temps, par des chercheurs finnois qui employaient dans ce cadre des collaborateurs de langue maternelle same, pour faire face aux obstacles culturels et linguistiques émergeant en cours de route.

1. A nyelvcserehelyzet olyan instabil többnyelvü helyzet, amelyben a közösség fokozatosan áttér az egyik nyelv használatáról egy másik nyelv használatára (vö. Weinreich 1953, Fishman 1968, Gal 1979). 


\section{RÉSUMÉS}

Les langues sames sont parlées dans quatre pays de Fennoscandie; ce sont des langues marginales et transfrontalières, qui bénéficient aujourd'hui du statut officiel de langues minoritaires et sont en général reconnues en tant que telles dans leur usage public. Les langues maternelles dans toutes les communautés sames sont considérées comme étant en danger. Dans ma thèse de doctorat, qui sert de base à cette étude, « Bilinguisme et attitudes linguistiques dans les communautés de Sames du Nord en Finlande»(2008), j'ai surtout analysé les facteurs extralinguistiques qui conduisent les communautés autogérées d'Enontekiö et de Sodankylä en Laponie finlandaise à changer progressivement de langue. J'ai montré par une étude sur questionnaire les corrélations entre usage de la langue et attitudes linguistiques. Dans la présente étude, je décris les normes d'utilisation de la langue qui caractérisent les membres de ces communautés, et je les mets en rapport avec d'autres variables - l'âge, le sexe, la profession et le niveau de formation. Les résultats montrent qu'aujourd'hui la communauté est indiscutablement en train de changer de langue, les différences de génération dans l'usage de la langue sont perceptibles: l'usage de la langue maternelle est réduit aux niveaux non formels, mais les pratiques ne sont pas en rapport direct avec les variables sociales énumérées ci-dessus.

A számi nyelvek Fenno-Skandinávia négy országában őshonos, határokon átnyúló peremnyelvek, amelyek ma hivatalos kisebbségi státussal rendelkeznek és nyilvános használatban többnyire elismert kisebbségi nyelvek. Valamennyi számi közösség anyanyelve a veszélyeztetett nyelvek közé, tagjai a nyelvcserehelyzetben lévő kisebbségi közösségekhez tartoznak ${ }^{1}$. A jelen tanulmány alapját képezo, „Kétnyelvűség és nyelvi attitűd finnországi északi számi beszélőközösségekben” (2008) című doktori disszertációmban elemeztem azokat az elsősorban nyelven kívüli tényezőket, amelyek Finnországban a lappföldi Enontekiö és Sodankylä önkormányzati területein élő északi számi közösségekben fokozatos nyelvváltást idéznek elő. A disszertációban egy kérdőíves vizsgálaton keresztül világítottam rá a nyelvhasználat és nyelvi attitűdök összefüggéseire. Jelen tanulmányban arra vállalkozom, hogy bemutatom a közösség tagjaira jellemző nyelvhasználati normákat, majd azokat összefüggésbe hozom az életkor, a nem, a foglalkozás és az iskolázottság változóival. Az eredmények azt mutatják, hogy a közösség ma egyértelműen nyelvcserehelyzetben van, a számi nyelvhasználat generációs megoszlásában különbségek észlelhetők, az anyanyelv használata visszaszorul az informális nyelvhasználati színterekre, és hogy a közösség tagjainak nyelvhasználati szokásai nem állnak szoros összefüggésben az említett társadalmi változókkal.

Sami is spoken in four Fennoscandian countries; the Sami are both a marginal and cross-border people who today have official minority language status, which is recognised as such in public use. In all Sami communities, mother tongues are considered to be endangered. In my doctoral dissertation, on which this study relies, Bilingualism and Linguistic Attitudes in Sami Communities in Northern Finland (2008), I mainly analysed extra-linguistic factors that lead selfmanaged Enontekiö and Sodankylä communities, in Finnish Lapland, to gradually change language. Using a questionnaire I showed correlations between language use and linguistic attitudes. In this study, I describe language use norms for community members and correlate them to other variables, such as age, gender, profession, and education. Results show that today the communities are changing language, and that a language use generation gap is to be felt: the use of the vernacular is limited to non-formal levels, but the practice is not directly correlated to the social variables listed above. 
INDEX

Thèmes : linguistique

disciplines same, same skolt, finnois, norvégien, suédois, russe

Index chronologique : XXIe siècle (début), XXIe siècle

nomsmotscles Finlandais, Sames

Mots-clés : situation linguistique, usage linguistique, bilinguisme, changement de langue

Index géographique : Finlande, Sodankylä, Utsjoki

Keywords : language situation, language use, Northern Sami, Bilingualism, language change 